\title{
Braucht Deutschland zusätzliche Kombilöhne?
}

Does Germany Need Additional In-work-benefits?

\section{Claudia Weinkopf ${ }^{*}$}

Soll man in Deutschland Kombilöhne einführen, um die Arbeitsanreize von Arbeitslosen zu erhöhen und mehr Beschäftigung im Niedriglohnbereich zu schaffen? Oder existieren schon diverse Regelungen mit Kombilohn-Charakter, die man ggf. weiter entwickeln und besser aufeinander abstimmen müsste? Welche Rolle könnte in diesem Kontext der Einführung eines Mindestlohnes zukommen? Die Bundesregierung hat im Koalitionsvertrag von September 2005 angekündigt, hierzu im Herbst 2006 ein Konzept zu entwickeln. In diesem Beitrag wird ausgehend von den bisherigen Erfahrungen mit Kombilohn-Modellprojekten dafür plädiert, an vorhandenen Ansätzen anzuknüpfen und diese ggf. zu modifizieren, aber sorgfältig zu prüfen, ob darüber hinaus zusätzliche Kombilöhne für spezielle Zielgruppen eingeführt werden sollten.

\section{Bisherige Erfahrungen}

Da es keine allgemein anerkannte Definition von Kombilöhnen gibt, ist das Spektrum der unter diesem Stichwort diskutierten Ansätze sehr breit und heterogen. Während in engeren Definitionen nur die Kombination von Arbeitseinkommen und Transfers an Beschäftigte als Kombilohn bezeichnet wird (Dietz et al. 2006: I), beziehen andere auch Zuschüsse ein, die auch oder sogar ausschließlich an Arbeitgeber gewährt werden, um Beschäftigung im Niedriglohnbereich zu fördern.

Die Debatte über Kombilöhne ist in Deutschland keineswegs neu. Vielmehr wurde bereits 1998/99 vor allem im Kontext des damaligen »Bündnis für Arbeit, Ausbildung und Wettbewerbsfähigkeit« sehr intensiv über die Einführung von Kombilöhnen diskutiert (vgl. Weinkopf 2006b). Letztlich wurde angeregt, zunächst zeitlich befristet und regional begrenzt unterschiedliche Varianten modellhaft zu erproben, was in der Praxis dann auch umgesetzt wurde - teilweise mit wissenschaftlicher Begleitung.

In Tabelle I (S. 202) sind die Fördermodalitäten von vier ausgewählten Ansätzen mit unterschiedlichen Zielgruppen und Subventionen zusammengestellt (vgl. ausführlicher z.B. Kaltenborn 200I). Das Mainzer Modell und das SGI-Modell wurden ab Mitte 2000 im Rahmen des Bundesprogramms "Chancen und Anreize zur Aufnahme sozialversicherungspflichtiger Tätigkeiten " (CAST) zunächst in ausgewählten Regionen umgesetzt; das Einstiegsgeld wurde in mehreren Kommunen und Kreisen in Baden-Württemberg erprobt. Das Hamburger Modell ist von der dortigen Arbeitsverwaltung entwickelt worden und ist der einzige Ansatz, dessen Umsetzung noch läuft.

Insgesamt war die Inanspruchnahme der Förderung in fast allen Modellprojekten und -programmen zur Erprobung von Kombilöhnen gering und blieb z.T. weit hinter den ur-

* Institut Arbeit und Technik, Gelsenkirchen. 


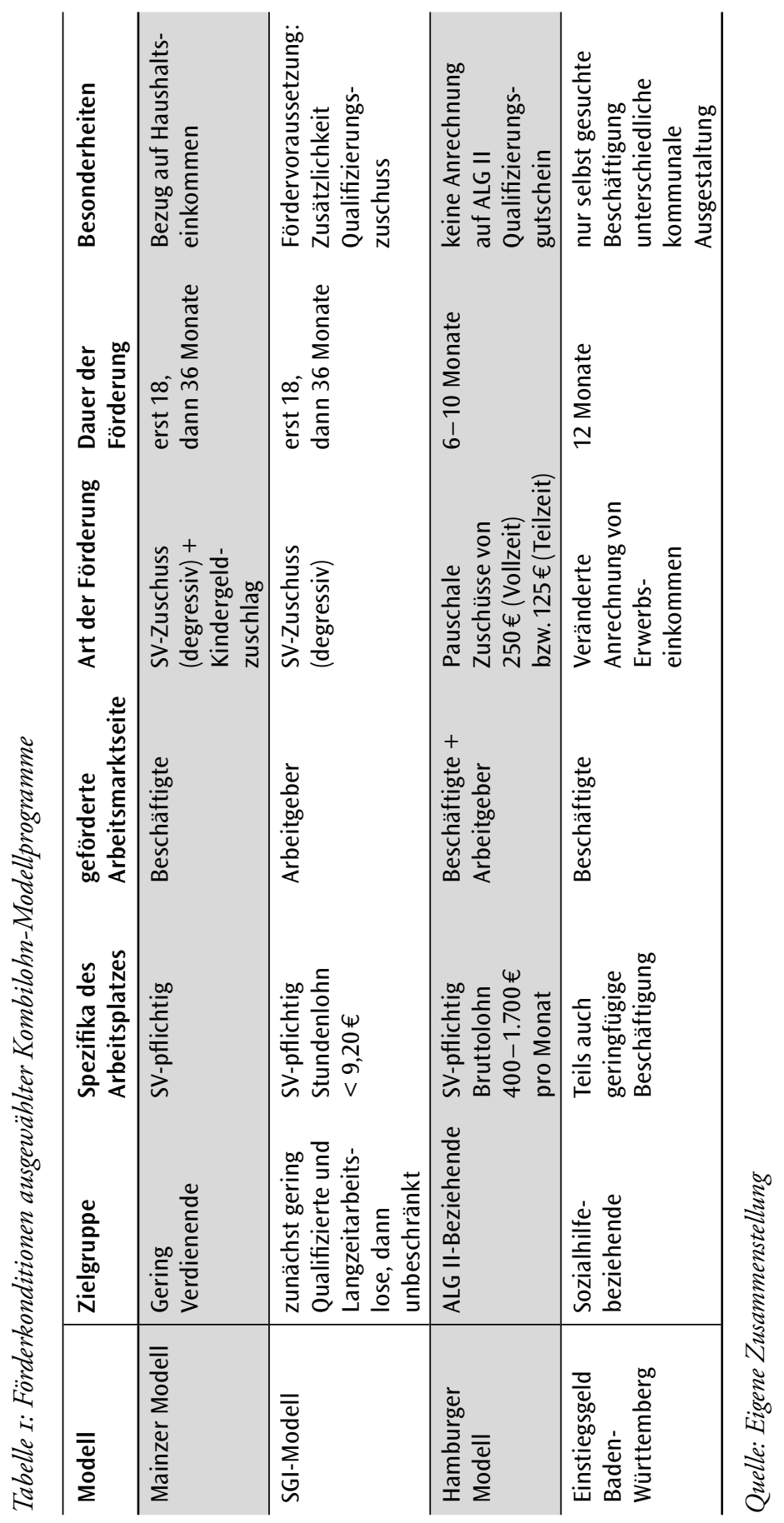


sprünglichen Erwartungen zurück. Der Zahl der Förderfälle der hier aufgeführten Modellprojekte lag zwischen 386 beim SGI-Modell, das deswegen auch vorzeitig eingestellt wurde, und I5.02I beim Mainzer Modell, als es 2002/2003 für ein Jahr bundesweit umgesetzt wurde. Das Einstiegsgeld in Baden-Württemberg wurde in 76I Fällen gewährt. Mit dem Hamburger Modell wurden demgegenüber zwischen März 2002 und Ende 2005 fast 8.000 Beschäftigungsverhältnisse gefördert, die allerdings im Durchschnitt von recht kurzer Dauer waren.

Die Zahl der Förderfälle ist nicht gleich zu setzen mit einem entsprechenden Zuwachs an Arbeitsplätzen. Dies liegt auch daran, dass die Einrichtung zusätzlicher Arbeitsplätze nur in Ausnahmefällen Voraussetzung für die Förderung war (z.B. beim SGI-Modell). Arbeitnehmer|innen|seitige Zuschüsse nach dem Mainzer Modell wurden hingegen gewährt, wenn eine Stelle mit einem Monatslohn unterhalb gewisser Einkommensgrenzen angenommen wurde und das Haushaltseinkommen bestimmte Grenzen nicht überstieg. Ob der Arbeitsplatz zusätzlich war oder die Einstellung ggf. auch ohne den Zuschuss erfolgt wäre, war ohne Belang. In vielen Fällen dürfte es sich um die Wiederbesetzung von Arbeitsplätzen im Zuge normaler Fluktuation gehandelt haben.

Einkommensbeihilfen bewirken grundsätzlich nur dann zusätzliche Beschäftigung, wenn der betreffende Arbeitsplatz ohne die Subvention nicht hätte besetzt werden können oder wenn Unternehmen mehr Arbeitsplätze anbieten, weil sie aufgrund der Subvention niedrigere Löhne durchsetzen können. Auch beim derzeit viel beachteten Hamburger Modell kann die Förderung im Rahmen der Fördergrenzen für jede sozialversicherungspflichtige Einstellung von Arbeitslosengeld II-Beziehenden in Anspruch genommen werden.

Zugunsten von Kombilöhnen wird häufig angeführt, dass sich hierdurch die Beschäftigungschancen von gering Qualifizierten verbessern ließen. Allerdings zeigen die Erfahrungen der Modellprojekte, dass hohe Anteile von gering Qualifizierten nur erreicht werden, wenn sich die Förderung weitestgehend auf diese Zielgruppe beschränkt, was etwa bei den NRW-Modellprojekten oder beim SGI-Modell der Fall war. Wird die Zielgruppewie bei den anderen hier betrachteten Modellprojekten - hingegen weiter gefasst, werden gering Qualifizierte offenbar seltener erreicht: Beim Einstiegsgeld in Baden-Württemberg verfügte etwa die Hälfte der Geförderten nicht über eine abgeschlossene Berufsausbildung (Dann et al. 2002: 43), beim Mainzer Modell lag der Anteil mit nur 36,6 Prozent noch deutlich niedriger (vgl. Kaltenborn et al. 2005).

Zur Stabilität von mit Kombilöhnen geförderten Arbeitsverhältnissen und zur Frage, ob sich die Beschäftigungsperspektiven der Betroffenen hierdurch (nachhaltig) verbessert haben, liegen kaum Erkenntnisse vor. Beim Mainzer Modell unterschied sich die Stabilität der geförderten Arbeitsverhältnisse in Ostdeutschland nicht von ungeförderter Beschäftigung, während sie in Westdeutschland sogar geringer war. In vielen Fällen wurden Arbeitsverhältnisse beendet, bevor die Förderhöchstdauer (zunächst I8 und später 36 Monate) erreicht war (vgl. Kaltenborn et al. 2005). Die Arbeitsverhältnisse beim Hamburger Modell dauern im Durchschnitt sogar nur drei bis vier Monate. Beim Einstiegsgeld in Baden-Württemberg und beim Hessischen Kombilohn konnte im Vergleich der geförderten Beschäftigten mit Kontrollgruppen nur z.T. ein positiver Effekt festgestellt werden (Dietz et al. 2006: 4). 
Zusammenfassend ist festzuhalten, dass die bisherigen Modellprojekte keinen Beweis erbracht haben, dass zeitlich befristete Kombilöhne zu einer Ausweitung der Beschäftigung (vgl. auch Kaltenborn 2003) oder zur nachhaltigen Verbesserung der Beschäftigungschancen benachteiligter Arbeitsloser beitragen können. Mit den Einkommensbeihilfen wurde immerhin erreicht, dass Personen mit geringen Arbeits- und oder Haushaltseinkommen zeitweilig finanziell besser gestellt wurden. Hiervon haben häufig insbesondere alleinerziehende Frauen profitiert, was zweifellos begrüßenswert ist. Allerdings stellt sich die Frage, ob eine höhere Erwerbsbeteiligung von Alleinerziehenden nicht auch mit einem Ausbau der Kinderbetreuung statt mit Kombilöhnen erreicht werden kann.

Ambivalent sind auch die Wirkungsanalysen unterschiedlicher Varianten von Kombilöhnen, die in anderen Ländern teilweise bereits seit langem existieren (vgl. ausführlicher Düll 2006, Jaehrling/Weinkopf 2006, Kaltenborn et al. 2006, Brücker/Konle-Seidl 2006). Die internationalen Erfahrungen verweisen darauf, dass großzügige Freibetragsregelungen oder Steuergutschriften am ehesten finanzierbar sind und Beschäftigungswirkung entfalten, wenn das Niveau der Mindestsicherung niedrig ist, womit aber das Ziel der Armutsvermeidung verletzt wird. Bei einem höheren Niveau der Transferleistungen an Arbeitslose führen niedrige Transferentzugsraten hingegen dazu, dass auch bereits Erwerbstätige bis in mittlere Einkommen hinein Anspruch auf Transferleistungen haben und die intendierte Steigerung der Arbeitsanreize begrenzt bleibt. Auch die Beschäftigungswirkungen arbeitgeberseitiger Zuschüsse zu den Sozialabgaben für Niedriglohnjobs, wie sie z. B. in Frankreich für Arbeitsplätze auf dem Niveau des gesetzlichen Mindestlohns bzw. leicht darüber gewährt werden, sind offenbar eher gering, obwohl die Kosten mit aktuell ca. I9 Milliarden $€$ pro Jahr keineswegs zu vernachlässigen sind (vgl. Brücker/Konle-Seidl 2006).

\section{Bereits existierende Kombilöhne in Deutschland}

Wenngleich aktuell in Deutschland oft über die »Einführung « von Kombilöhnen diskutiert wird, gibt es bereits heute eine Reihe von Regelungen, die eine Kombination von Transferleistungen und Arbeitseinkommen ermöglichen und insofern als bereits existierende arbeitnehmerseitige Kombilöhne anzusehen sind. Neben zeitlich begrenzten und auf bestimmte Gruppen beschränkten Ansätzen, wie etwa das Einstiegsgeld im SGB II, der Kinderzuschlag und die Entgeltsicherung für Ältere (vgl. ausführlicher Jaehrling/Weinkopf 2006), betrifft dies vor allem die Möglichkeit, bei niedrigen Einkommen ergänzende Leistungen der Grundsicherung (Arbeitslosengeld II) zu beziehen, und die Sonderregelungen für Mini- und Midijobs.

Nachdem die Bundesagentur für Arbeit im März 2006 erstmals Daten zur Zahl der Bedarfsgemeinschaften bzw. Personen veröffentlicht hat, die über Erwerbseinkommen verfügten, aber gleichzeitig Anspruch auf ergänzende Leistungen der Grundsicherung hatten, ist deutlich geworden, dass dies im September 2005 etwa jede|n Fünfte|n betraf. Die absolute Zahl der Erwerbstätigen in Bedarfsgemeinschaften lag bei 906.00o Personen. Von diesen erzielte gut die Hälfte ( 5 I Prozent) ein Bruttoeinkommen unterhalb der Geringfügigkeitsgrenze von $400 €$, I9 Prozent zwischen 400 und $800 €$ und 30 Prozent mehr als $800 €$ 
(Bundesagentur für Arbeit 2006: 4). Zu welchen Anteilen es sich hierbei um Personen handelte, die aus der Arbeitslosigkeit heraus eine niedrig bezahlte Erwerbstätigkeit aufgenommen haben, bzw. um bereits zuvor Erwerbstätige, die ein Arbeitseinkommen erzielen, das nicht ausreicht, um den Lebensunterhalt zu bestreiten, ist bislang nicht bekannt. Da rund ein Drittel der erwerbstätigen ALG II-Beziehenden ein anrechenbares Einkommen von unter $200 €$ erzielten, wird häufig angenommen, dass diese sich hierbei an den Grenzen des anrechnungsfreien Zuverdienstes orientieren (Grabowsky et al. 2006: 68). Allerdings lag der Anteil derjenigen, die trotz Vollzeitarbeit Anspruch auf ergänzende Leistungen der Grundsicherung hatten, nur unwesentlich niedriger.

Vor diesem Hintergrund erscheint es unangemessen, pauschal zu unterstellen, dass Arbeitslose danach streben, die Kombination aus Arbeitseinkommen und Sozialleistungen zu optimieren, was häufig als Missbrauch bezeichnet wird. Dass auch auf Seiten der Unternehmen eine missbräuchliche Nutzung der Grundsicherung vorkommen kann - etwa, indem Löhne zulasten ergänzender ALG II-Leistungen abgesenkt werden -, wird hingegen kaum thematisiert. Vernachlässigt wird dabei auch, dass Niedriglohnbeschäftigung in Deutschland keineswegs erst mit der Umsetzung von Hartz IV an Bedeutung gewonnen hat und dass es sich keineswegs nur um Zuverdiener|innen handelt, sondern in erheblichem Umfang auch Vollzeitbeschäftigte betrifft (vgl. Kalina/Weinkopf 2006). Darüber hinaus haben Teilzeitarbeit und Minijobs in den vergangenen Jahren zugenommen, aus deren Verdienst selbst bei höheren Stundenlöhnen eine eigenständige Existenzsicherung häufig nicht möglich ist. Dies geht keineswegs alleine auf Wünsche der Beschäftigten nach kurzen Arbeitszeiten zurück, sondern ist vor allem in einigen Dienstleistungsbranchen Teil unternehmerischer Strategien, die auf eine kostengünstige Optimierung des Personaleinsatzes zielen. So sind inzwischen z.B. in der Gebäudereinigung nur noch rund 25 Prozent aller Arbeitsplätze sozialversicherungspflichtige Vollzeitstellen (vgl. Hieming et al. 2005).

Die Politik hat diese Entwicklung durch die Reform der geringfügigen Beschäftigung im Frühjahr 2003, durch die Mini- und Midijobs eingeführt wurden, mit befördert. Inzwischen liegt die Zahl der Minijobs bei deutlich über sechs Millionen; die Zahl der Midijobs wurde für Ende 2003 auf rund 670.000 beziffert. Aufgrund der Sonderregelungen, die für diese Arbeitsverhältnisse im Steuer- bzw. Sozialversicherungsrecht gelten, sind auch sie als spezifische Form von »Kombilöhnen " anzusehen, wenngleich kein direkter materieller Zuschuss (Transfer) an die Beschäftigten geleistet wird, sondern die Vergünstigung darin besteht, dass keine oder reduzierte Sozialabgaben erhoben werden und der Verdienst aus einem Minijob grundsätzlich steuerfrei ist. Das Haushaltseinkommen (oder bei Nebenbeschäftigten der Verdienst aus dem Hauptjob) spielt hierbei keine Rolle. Bei Midijobs mit einem monatlichen Bruttoeinkommen über $400 €$ bis zu $800 €$ fallen arbeitnehmer|innen|seitig nur ermäBigte, aber mit dem Einkommen steigende Sozialversicherungsbeiträge an (Gleitzone), während zumindest in der Kranken- und Pflegeversicherung volle Leistungsansprüche bestehen. Auch hier gibt es keine Beschränkungen dieser Vergünstigungen auf bestimmte Zielgruppen oder einen Bezug zum Haushaltseinkommen.

Wenngleich Unternehmen für Midijobs den normalen Beitrag zur Sozialversicherung und für Minijobs sogar einen erhöhten Pauschalsatz von 25 Prozent (seit Juli 2006: 30 Pro- 
zent) abführen müssen, gibt es Hinweise, dass auch sie vor allem mit der Nutzung von Minijobs Kostenvorteile realisieren: So liegen die Stundenlöhne bei über 85 Prozent der Minijobs unterhalb der Niedriglohnschwelle, was darauf hindeutet, dass vielfach Lohnabschläge realisiert werden. Weiterhin werden geringfügig Beschäftigten offenbar häufig betriebliche Leistungen wie etwa Lohnfortzahlung im Krankheitsfall vorenthalten (vgl. Kalina/Weinkopf 2006). Ein weiterer Vorteil für Unternehmen besteht darin, dass sie mit Minijobs häufig auch Personengruppen erreichen, die höhere Qualifikationen und Sozialkompetenzen mitbringen, ohne dass diese angemessen entlohnt werden müssen - z.B. Studierende, Rentner|innen, Nebenbeschäftigte und Wiedereinsteiger|innen, die aus unterschiedlichen Gründen eine kurze Arbeitszeit bevorzugen und den Großteil der geringfügig Beschäftigten stellen.

\section{Schlussfolgerungen}

Angesichts der großen Zahl von Menschen, die bereits heute in der einen oder anderen Form Kombilöhne beziehen, erscheint es fraglich, ob Deutschland darüber hinaus weitere Kombilöhne braucht. Aus unserer Sicht spricht vieles dafür, die bestehenden Regelungen der Grundsicherung weiter zu entwickeln, statt völlig neue Kombilöhne einzuführen. Welche Ansatzpunkte hierbei bestehen und welche Probleme und Risiken dabei auftreten können, wird im Folgenden skizziert.

Das neue System der Grundsicherung bietet offenbar nicht nur eine finanzielle Mindestabsicherung für Arbeitslose, sondern erfüllt auch die Funktion, niedrige Erwerbseinkommen im Haushaltskontext bedarfsgeprüft aufzustocken. Kritisiert wird hieran insbesondere, dass verstärkte Eigenbemühungen zur Bestreitung des Lebensunterhaltes aufgrund hoher Transferentzugsraten nur unzureichend honoriert werden. Die Unterstellung, dass viele Personen mit Anspruch auf ergänzende Leistungen der Grundsicherung den Umfang ihrer Erwerbstätigkeit bewusst so ausgestalten, dass mit begrenztem Aufwand das verfügbare Einkommen optimiert wird, verkennt gleichwohl, dass die Art der verfügbaren Erwerbsmöglichkeiten auch entscheidend dadurch geprägt wird, welche Arbeitsplätze Unternehmen anbieten.

Ungeachtet dieser Einschränkung besteht die Möglichkeit, die Transferentzugsraten für Erwerbseinkommen insgesamt zu verringern oder so zu verändern, dass insbesondere die Anreize zur Ausübung einer mehr als geringfügigen Beschäftigung gestärkt werden. Letzteres könnte allerdings dazu führen, dass kleinere Nebenjobs verstärkt in Schwarzarbeit ausgeübt würden. Insgesamt niedrigere Transferentzugsraten haben den Nachteil, dass sich hierdurch die Einkommensgrenzen, bis zu denen Anspruch auf ergänzende Leistungen der Grundsicherung besteht, nach oben verschieben. Der Kreis der Anspruchsberechtigten würde größer, was im Ergebnis ggf. sogar zu einem höheren finanziellen Aufwand führen könnte. Vermieden werden könnte dieser Effekt ggf. dadurch, dass verringerte Transferentzugsraten nur zeitlich befristet gewährt würden (vgl. Spermann 2006).

Um die finanziellen Spielräume für geringere Transferentzugsraten bzw. höhere Freibeträge für Erwerbseinkommen nach den angelsächsischen Vorbildern zu vergrößern, wird 
oftmals gefordert, die Transferleistungen für Erwerbsfähige abzusenken, wie dies auch im ifo-Konzept der "Aktivierenden Sozialhilfe« vorgesehen ist (vgl. Sinn et al. 2006). Da eine Absenkung des Niveaus der Transferleistungen in Deutschland jedoch verfassungsrechtlich problematisch erscheint, müssten für arbeitsfähige Arbeitslose, die auf dem ersten Arbeitsmarkt keine Beschäftigung finden, öffentliche Beschäftigungsangebote organisiert werden. Nach dem ifo-Konzept wäre deren Annahme obligatorisch, um Anspruch auf die volle Grundsicherung zu erhalten.

Jenseits sozialpolitischer Risiken und Bedenken, die hier nicht weiter ausgeführt werden können (vgl. Weinkopf 2006a), könnte die erforderliche massenhafte Bereitstellung öffentlicher Beschäftigungsmöglichkeiten jedoch zu einer Reihe von Problemen führen. So wären die Kosten und der Organisationsaufwand auch angesichts der hohen Fluktuation von Niedriglohnbeschäftigten voraussichtlich sehr hoch. Bei erneuter Arbeitslosigkeit müsste jeweils kurzfristig eine kommunale Arbeitsmöglichkeit bereitgestellt werden. Das Ausmaß der öffentlichen Ersatzbeschäftigung würde zudem bei weitem die heutige Größenordnung der so genannten Ein-Euro-Jobs übersteigen, so dass enorme Verdrängungseffekte auftreten könnten. Nicht zuletzt würde die intendierte erhebliche Absenkung des Lohnniveaus voraussichtlich dazu führen, dass die Zahl der Beschäftigten, die Anspruch auf ergänzende Grundsicherung bzw. Steuergutschriften hätten, stark anstiege, was erhebliche finanzielle Risiken birgt.

Eine gänzlich andere »Stellschraube« zur Reduzierung des finanziellen Aufwandes für die Aufstockung von niedrigen Erwerbseinkommen, die nicht am Niveau der Transferleistungen ansetzt, wäre die Einführung eines gesetzlichen Mindestlohnes, um eine untere Grenze für staatliche Zuzahlungen zu niedrigen Löhnen einzuziehen. In Großbritannien hat dieses Argument bei der Einführung des gesetzlichen Mindestlohns im Jahre I999 eine wichtige Rolle gespielt: Anderenfalls bestehe die Gefahr, dass Unternehmen Niedriglöhne weiter absenken würden, weil sie sich darauf verlassen könnten, dass der Staat die Ausfallbürgschaft übernehme (vgl. Bosch / Weinkopf 2006). Die deutlichen Erhöhungen des britischen Mindestlohns in den vergangenen Jahren haben hier Spielräume geschaffen, um die Einkommensgrenzen für Steuergutschriften anzuheben und auf Kinderlose auszuweiten.

Eine weitere Möglichkeit besteht darin, Arbeitslose über eine Intensivierung des »Förderns« stärker zu aktivieren. Dies gelänge allerdings am besten, wenn auch entsprechende Stellenangebote vorhanden wären. Derzeit haben Unternehmen jedoch offenbar oftmals kaum Schwierigkeiten, auch niedrig bezahlte Stellen zu besetzen, und verzichten häufig auf deren Meldung bei der öffentlichen Arbeitsvermittlung (vgl. Hieming et al. 2005).

Ergänzend zu den hier aufgezeigten unterschiedlichen Reformmöglichkeiten der Grundsicherung wäre die Ausgestaltung der Mini- und Midijobs auf den Prüfstand zu stellen. Insbesondere die Minijobs sind zwar bei Unternehmen wie Beschäftigten sehr beliebt, aber es erscheint mehr als fraglich, ob die Anreize zur Aufspaltung von sozialversicherungspflichtigen Beschäftigungsverhältnissen nicht deutlich verringert bzw. beseitigt werden müssten. Denn diese führen zu erheblichen Einnahmeverlusten in der Sozialversicherung und dazu, dass andere Personengruppen bei Einstellungen Arbeitslosen oftmals vorgezogen werden. 
Ob über die bestehenden Regelungen hinaus zusätzliche Kombilöhne für spezielle Zielgruppen eingeführt werden sollten, sollte sorgfältig geprüft werden. Hierbei ist einerseits zu berücksichtigen, ob bereits Förderinstrumente für die jeweilige Zielgruppe existieren, die ggf. in Konkurrenz zu einem neuen Kombilohn treten könnten. Andererseits ist zu analysieren, welche Art der Förderung am besten geeignet erscheint, um die Beschäftigungshemmnisse der jeweiligen Zielgruppe zu verringern. Auch die Frage der Begrenzung von Mitnahmeund Verdrängungseffekten verdient besondere Aufmerksamkeit. Und nicht zuletzt sollten flankierende Maßnahmen vorgesehen werden, um den Übergang der Beschäftigten in ungeförderte Arbeit zu unterstützen. Anderenfalls könnten Niedriglohnjobs zunehmend zur Sackgasse werden.

\section{Literatur}

Bosch, Gerhard/Weinkopf, Claudia (2006): Mindestlöhne in Großbritannien - ein geglücktes Realexperiment, in: WSI-Mitteilungen, Jg. 59, H. 3, S. I25-130

Brücker, Herbert/ Konle-Seidl, Regina (2006): Kombilöhne im internationalen Vergleich. Nicht jede Therapie schlägt überall an, IAB-Kurzbericht, Nr. Io

Bundesagentur für Arbeit (2006): Grundsicherung für Arbeitsuchende: Anrechenbare Einkommen und Erwerbstätigkeit, Nürnberg

Dann, Sabine / Kirchmann, Andrea / Spermann, Alexander/Volkert, Jürgen (2002): Einstiegsgeld in Baden-Württemberg. Schlussbericht, herausgegeben vom Sozialministerium BadenWürttemberg, Stuttgart

Dietz, Martin / Koch, Susanne/Walwei, Ulrich (2006): Kombilöhne. Ein Ansatz mit Haken und Ösen, IAB-Kurzbericht, Nr. 3

Düll, Herbert (2006): Arbeitsanreize im Kontext des Steuer- und Transfer-Systems: Ein ausgewählter internationaler Vergleich, in: Bundesarbeitsblatt, Nr. 4, S. 4-16

Grabowsky, Fabian/Neubacher, Alexander/Sauga, Michael (2006): Die große Flut, in: Der Spiegel vom 08.05.2006, Nr. 19, S. 66-68

Hieming, Bettina/Jaehrling, Karen / Kalina, Thorsten/Vanselow, Achim/Weinkopf, Claudia (2005): Stellenbesetzung im Bereich »einfacher« Dienstleistungen. Abschlussbericht einer Studie im Auftrag des Bundesministeriums für Wirtschaft und Arbeit, BMWADokumentation, Nr. 550, Berlin

Jaehrling, Karen/Weinkopf, Claudia (unter Mitarbeit von Bettina Hieming und Bruno Kaltenborn) (2006): Kombilöhne in Deutschland - neue Wege, alte Pfade, Irrweg?, Expertise im Auftrag der Friedrich-Ebert-Stiftung, Gelsenkirchen, i.E.

Kalina, Thorsten / Weinkopf, Claudia (2006): Mindestens 6 Millionen Niedriglohnbeschäftigte in Deutschland: Welche Rolle spielen Teilzeitarbeit und Minijobs?, IAT-Report, Nr. 2006-03

Kaltenborn, Bruno (200I): Kombilöhne in Deutschland - eine systematische Übersicht, IABWerkstattbericht, Nr. I4

Kaltenborn, Bruno (2003): Kombilohn: Stand und Perspektiven, in: DIW Vierteljahrshefte zur Wirtschaftsforschung, Jg. 72, H. I, S. I24-I32 
Kaltenborn, Bruno / Krug, Gerhard / Rudolph, Helmut/Weinkopf, Claudia / Wiedemann, Eberhard (2005): Evaluierung des arbeitsmarktpolitischen Sonderprogramms CAST. Endbericht des Forschungsverbundes, BMWA-Forschungsbericht, Nr. 552, Berlin

Kaltenborn, Bruno/Schiwarov, Julia/Wielage, Nina (2006): Arbeitsanreize im Niedriglohnbereich - ein internationaler empirischer Überblick, Beiträge zur Wirtschaftsforschung und Politikberatung, Nr. 3I, Berlin

Sinn, Hans-Werner / Holzner, Christian / Meister, Wolfgang/ Ochel, Wolfgang/Werding, Martin (2006): Aktivierende Sozialhilfe 2006: Das Kombilohn-Modell des ifo Instituts, in: ifoSchnelldienst, Jg. 59, H. 2, S. 3-24

Spermann, Alexander (2006): Mehr Druck, mehr Anreiz. Wie ein erfolgreiches KombilohnModell aussehen könnte, in: Die ZEIT vom I9.0I.2006

Weinkopf, Claudia (2006a): Kombilöhne - Kein Patentrezept für den Arbeitsmarkt, in: Soziale Sicherheit, Jg. 55, H. 3, S. 98-102

Weinkopf, Claudia (2006b): Was bringen Kombilöhne?, in: Sterkel, Gabriele/ Schulten, Thorsten/Wiedemuth, Jörg (Hg.), Mit Mindestlöhnen gegen Sozialdumping, Hamburg, S. $80-103$

\section{Inequality and Efficiency - Does a Wider Spread of Wages Raise Employment? Ronald Schettkat*}

\section{A Unified Theory?}

The assumed trade-off between efficiency and equality is deeply engraved in the minds of many economists. Applied to labor markets this assertion says that a wider dispersion of wages raises employment. Welch (1999), addressing the American Economic Association in the Richard T. Ely Lecture`, praises the beauty of an economy with high inequality, and sociologists claim that inequality is the price for freedom (Dahrendorf 2006). Why should a wider wage dispersion improve employment? Many hypotheses have been forwarded:

I. wages equal workers' marginal productivity and therefore a wider dispersion of wages allows for an integration of the less productive workers;

2. workers of all ranks are - like tournament players - motivated by the high rewards for the winner, i.e. the top earners;

3. all western economies were hit by the same shocks (skill-biased technological change, globalization), which are disadvantageous for less skilled workers and result in a wider

* University of Wuppertal. 